PURD-TH-95-02

\title{
The Supercurrent In Supersymmetric Field Theories
}

\author{
T.E. Clark and S.T. Love \\ Department of Physics \\ Purdue University \\ West Lafayette, IN 47907-1396
}

\begin{abstract}
A supercurrent superfield whose components include a conserved energymomentum tensor and supersymmetry current as well as a (generally broken) $\mathrm{R}$-symmetry current is constructed for a generic effective $\mathrm{N}=1$ supersymmetric gauge theory. The general form of the R-symmetry breaking is isolated. Included within the various special cases considered is the identification of those models which exhibit an unbroken R-symmetry. One such example corresponds to a non-linearly realized gauge symmetry where the chiral field $\mathrm{R}$-weight is required to vanish.
\end{abstract}




\section{Introduction}

The most general graded Lie algebra of symmetries of the S-matrix of a relativistic quantum field theory is the direct product of (extended) supersymmetry (SUSY) with some internal symmetry [1]. That is, supersymmetry is the only possible extension of the Poincaré space-time symmetries. Moreover, since models possessing supersymmetry tend to exhibit a less singular ultraviolet behavior than what would naively be expected, one is naturally led to explore the role of SUSY in possible extensions of general relativity and quantum theories of gravity. Indeed, supersymmetry plays a pivotal role in many of the string theories [2] which offer the promise of incorporating gravity in a consistent quantum mechanical framework.

This softer ultraviolet behavior of supersymmetric theories can be encoded in various non-renormalization theorems [3], which, among other things, guarantees that supersymmetric models are free of additive quadratic divergences even when they contain fundamental scalar degrees of freedom. This attribute allows mass hiearchies which are established at tree level in such theories to remain stable against quantum fluctuations and has led to a considerable amount of activity in SUSY model building [4. Such a SUSY effective theory often arises as the flat space-time limit of a some supergravity model which in turn can be considered as the zero slope limit of an underlying superstring theory. The resulting SUSY model will, in general, contain interaction terms beyond those appearing in the perturbatively renormalizable case.

It has also been demonstrated [5] [6] that the restrictions imposed by SUSY may dictate that certain exact results can be established even after the inclusion of perturbative and nonperturbative radiative corrections. A cru- 
cial ingredient used in securing these results involves the (extended) SUSY algebra [0] which, in turn, is related to the supersymmetry currents. In addition, explorations [8] continue into the possibility of having non-perturbative violations of the non-renormalization theorems and dynamical supersymmetry breaking which in turn could provide for the natural origin of the huge hierarchy between the Planck scale and the electroweak scale. It has been argued that the nature of the R-symmetry realization plays an important role in determining the viability and calculability of this potentiality. Once again, the resultant SUSY models are generally required to contain higher dimensional operators in order to secure a stable ground state.

For perturbatively renormalizable models containing Yang-Mills vector superfields and (anti-) chiral superfields, it has been shown that the supersymmetry current is intimately related to the energy-momentum tensor and the R-symmetry current. In fact, a supercurrent multiplet [9] can be constructed such that its components contain these currents. Futhermore, the generalized (spinor) trace of the supercurrent not only describes the (non-) conservation of these component currents, but also that of the associated superconformal symmetry currents. Since, at the present time, many of the supersymmetric models being investigated involve more general structures than those appearing in this perturbatively renormalizable class, we constuct, in this paper, the general form of the supercurrent in a larger class of models characterized by arbitrary superpotential and prepotential functions as well as an arbitrary Kähler potential.

In the next section, we define the model action which is the general supersymmetric and gauge invariant form containing at most two derivatives. We also introduce functional differential operator representations for both the in- 
ternal gauge symmetry, which can be either linearly or non-linearly realized, and the space-time Poincaré, supersymmetry and R- transformations. Starting with the supercurrent trace identity, section 3 details the construction of the supercurrent which is secured by combining the various space-time symmetries into a particular superfield structure with the R-symmetry current as the lowest component. So doing, we obtain the general form of possible $\mathrm{R}$-symmetry breaking. As a special case, we review the form of the supercurrent obtained in perturbatively renormalizable SUSY models. In addition, we delineate the general criterion nessecary for an unbroken R-symmetry. 


\section{The Supersymmetric and Gauge Invariant Action}

Through two derivatives, the most general supersymmetric and gauge invariant action, $\Gamma$, composed of Yang-Mills vector superfields, $V^{A}$, and matter (anti-) chiral superfields, $\left(\bar{\phi}^{\bar{i}}\right) \phi^{i}$, which transform either linearly or nonlinearly under the gauge group $\mathrm{G}$, is

$$
\begin{gathered}
\Gamma[\phi, \bar{\phi}, V]=\int d V K(\phi, \bar{\phi}, V)+\int d S\left[\frac{1}{2} f_{A B}(\phi) W^{A \alpha} W_{\alpha}^{B}+P(\phi)\right] \\
+\int d \bar{S}\left[\frac{1}{2} \bar{f}_{A B}(\bar{\phi}) \bar{W}_{\dot{\alpha}}^{A} \bar{W}^{B \dot{\alpha}}+\bar{P}(\bar{\phi})\right] .
\end{gathered}
$$

This action contains a locally invariant Kähler potential [10], $K=K(\phi, \bar{\phi}, V)$, the SUSY Yang-Mills kinetic term multiplying (anti-) chiral field dependent prepotential functions, $\left(\bar{f}_{A B}(\bar{\phi})\right) f_{A B}(\phi)$, and the (anti-) chiral superpotential $(\bar{P}(\bar{\phi})) P(\phi)$. The adjoint representation chiral spinor field strength $W_{\alpha}$ 111 is

$$
W_{\alpha} \equiv W_{\alpha}^{A} t^{A}=-\frac{1}{4} \bar{D} \bar{D}\left[e^{-2 V} D_{\alpha} e^{2 V}\right]
$$

where $t^{A}$ are the adjoint representation matrices, $\left(t^{A}\right)_{B C} \equiv i f_{B A C}$ and

$V \equiv t^{A} V^{A}$ is the matrix valued gauge field. It proves convenient to introduce the polynomial in $V$ combination

$$
\ell_{A B} \equiv\left(\frac{e^{2 V}-1}{V}\right)_{A B}
$$

in terms of which we can write

$$
W_{\alpha}=-\frac{1}{4} \bar{D} \bar{D}\left[D_{\alpha} V^{B} \ell_{B A}\right] t^{A}
$$

which explicitly identifies the $W_{\alpha}^{A}$ spinors as

$$
W_{\alpha}^{A}=-\frac{1}{4} \bar{D} \bar{D}\left[D_{\alpha} V^{B} \ell_{B A}\right] .
$$


Similarly the anti-chiral field strength is

$$
\begin{aligned}
\bar{W}_{\dot{\alpha}} & =\bar{W}_{\dot{\alpha}}^{A} t^{A}=-\frac{1}{4} D D\left[e^{2 V} \bar{D}_{\dot{\alpha}} e^{-2 V}\right] \\
& =-\frac{1}{4} D D\left[\bar{D}_{\dot{\alpha}} V^{B} \bar{\ell}_{B A}\right] t^{A}
\end{aligned}
$$

where

$$
\bar{\ell}_{A B}=\left(\frac{e^{-2 V}-1}{V}\right)_{A B}=-\ell_{B A},
$$

and

$$
\bar{W}_{\dot{\alpha}}^{A}=-\frac{1}{4} D D\left[\bar{D}_{\dot{\alpha}} V^{B} \bar{\ell}_{B A}\right]
$$

\subsection{Gauge Invariance}

The generators of the symmetry groups can be realized using Ward identity functional differential operators acting on the superfields. The infinitesimal gauge transformations of the fields are defined by the functional differential operator

$$
\begin{array}{r}
\delta(\Lambda, \bar{\Lambda}) \equiv \int d S \Lambda^{A} A_{A}^{i}(\phi) \frac{\delta}{\delta \phi^{i}}+\int d \bar{S} \bar{\Lambda}^{A} \bar{A}_{A}^{\bar{i}}(\bar{\phi}) \frac{\delta}{\delta \overline{\phi^{i}}} \\
-i \int d V\left(\Lambda^{B} \ell_{B A}^{-1}+\bar{\Lambda}^{B} \bar{\ell}_{B A}^{-1}\right) \frac{\delta}{\delta V^{A}}
\end{array}
$$

where $\left(\bar{\Lambda}^{A}\right) \Lambda^{A}$ are the infinitesimal (anti-) chiral superfields parameterizing the gauge variation. When applied directly to the fields themselves, this yields their individual variations as

$$
\begin{aligned}
\delta(\Lambda, \bar{\Lambda}) \phi^{i} & =\Lambda^{A} A_{A}^{i}(\phi) \\
\delta(\Lambda, \bar{\Lambda}) \bar{\phi}^{\bar{i}} & =\bar{\Lambda}^{A} \bar{A}_{A}^{\bar{i}}(\bar{\phi}) \\
\delta(\Lambda, \bar{\Lambda}) V^{A} & =-i\left[\Lambda^{B} \ell_{B A}^{-1}+\bar{\Lambda}^{B} \bar{\ell}_{B A}^{-1}\right] .
\end{aligned}
$$


The (chiral) Killing vectors $A_{A}^{i}(\phi)$ and their (anti-chiral) complex conjugates, $\bar{A}_{A}^{\bar{i}}(\bar{\phi})$, define the global transformations of the matter fields, which are denoted by the variation $\delta_{A}$, so that

$$
\begin{aligned}
\delta_{A} \phi^{i} & =A_{A}^{i}(\phi) \\
\delta_{A} \bar{\phi}^{\bar{i}} & =\bar{A}_{A}^{\bar{i}}(\bar{\phi}) .
\end{aligned}
$$

These (anti-) chiral Killing vectors obey their defining Lie derivative or Killing equations

$$
\begin{aligned}
A_{A}^{j} A_{B}^{i}, j-A_{B}^{j} A_{A}^{i},{ }_{j} & =i f_{A B C} A_{C}^{i} \\
\bar{A}_{A}^{\bar{j}} \bar{A}_{B}^{\bar{i}}, \bar{j}-\bar{A}_{B}^{\bar{j}} \bar{A}_{A}^{\bar{i}}, \bar{j} & =i f_{A B C} \bar{A}_{C}^{\bar{i}},
\end{aligned}
$$

where we have introduced a notation where subscripts following commas denote differentiation so that, for example, $A_{B}^{i},{ }_{j}=\frac{\partial A_{B}^{i}}{\partial \phi^{j}}, \bar{A}_{B}^{\bar{i}}, \bar{j}=\frac{\partial \bar{A}_{B}^{i}}{\partial \bar{\phi}^{j}}$. These equations are a direct consequence of the gauge transformation algebra

$$
\left[\delta(\Lambda, \bar{\Lambda}), \delta\left(\Lambda^{\prime}, \bar{\Lambda}^{\prime}\right)\right]=i \delta\left(\Lambda \times \Lambda^{\prime}, \bar{\Lambda} \times \bar{\Lambda}^{\prime}\right),
$$

where the cross product is defined by the totally antisymmetric structure constant of the group, $f_{A B C}$, so that $\left(\Lambda \times \Lambda^{\prime}\right)_{A}=f_{A B C} \Lambda^{B} \Lambda^{\prime C}$.

For linear realizations of the gauge symmetry, Eq. (2.12) is solved by $A_{A}^{i}=i\left(T^{A}\right)_{i j} \phi^{j}$, where the $T^{A}$ form a matrix representation (perhaps reducible) of the group so that $\left[T^{A}, T^{B}\right]=i f_{A B C} T^{C}$. On the other hand, for non-linear realizations, such as in the case of supersymmetric non-linear sigma models [10] 12] [13], the $A_{A}^{i}$ solving Eq. (2.12) and thus forming a realization of the algebra are non-linear functions of the $\phi^{i}$. Using the local $\phi^{i}$ transformation law, the superpotential, $P(\phi)$, is seen to be locally invariant, $\delta(\Lambda, \bar{\Lambda}) P(\phi)=\Lambda^{A} A_{A}^{i}(\phi) P(\phi),{ }_{i}=0$, provided it is globally invariant, $\delta_{A} P(\phi)=A_{A}^{i}(\phi) P(\phi)_{, i}=0$. 
The gauge group transformation of the Yang-Mills vector superfields is defined via

$$
e^{2 V^{\prime}}=e^{2(V+\delta V)} \equiv e^{-i \Lambda} e^{2 V} e^{+i \bar{\Lambda}}
$$

For infinitesimal $\Lambda$ and $\bar{\Lambda}$, this reduces, upon application of the BakerCampbell-Hausdorff formula, to

$$
\delta(\Lambda, \bar{\Lambda}) V^{A}=\frac{1}{2}\left(\bar{\Lambda}^{B}+\Lambda^{B}\right) f_{A B C} V^{C}+\frac{i}{2}\left(\bar{\Lambda}^{B}-\Lambda^{B}\right)[V \operatorname{coth} V]_{B A},
$$

which can be shown to be identical to the last line of Eq. (2.10). Since this result is also consistent with the group algebra (Eq. (2.13)), this transformation also forms a realization of the gauge group. Using the vector field gauge transformation, it is readily established that the field strength spinors transform as the adjoint representation under gauge transformations:

$$
\begin{aligned}
\delta(\Lambda, \bar{\Lambda}) W_{\alpha} & =i\left[\Lambda, W_{\alpha}\right] \\
\delta(\Lambda, \bar{\Lambda}) \bar{W}_{\dot{\alpha}} & =i\left[\bar{\Lambda}, \bar{W}_{\dot{\alpha}}\right],
\end{aligned}
$$

or equivalently

$$
\begin{aligned}
\delta(\Lambda, \bar{\Lambda}) W_{\alpha}^{A} & =i\left(\Lambda^{C} t^{C}\right)_{A B} W_{\alpha}^{B} \\
\delta(\Lambda, \bar{\Lambda}) \bar{W}_{\dot{\alpha}}^{A} & =i\left(\bar{\Lambda}^{C} t^{C}\right)_{A B} \bar{W}_{\dot{\alpha}}^{B} .
\end{aligned}
$$

Since any non-trivial (anti-) chiral prepotential terms, $\left(\bar{f}_{A B}(\bar{\phi})\right) f_{A B}(\phi)$ are constructed to transform as the product of the (anti-) chiral adjoint representations of the gauge group so that,

$$
\begin{aligned}
\delta(\Lambda, \bar{\Lambda}) f_{A B} & =i\left(\Lambda^{D} t^{D}\right)_{A C} f_{C B}+i\left(\Lambda^{D} t^{D}\right)_{B C} f_{A C} \\
\delta(\Lambda, \bar{\Lambda}) \bar{f}_{A B} & =i\left(\bar{\Lambda}^{D} t^{D}\right)_{A C} \bar{f}_{C B}+i\left(\bar{\Lambda}^{D} t^{D}\right)_{B C} \bar{f}_{A C},
\end{aligned}
$$

it follows that the contractions $W^{A \alpha} f_{A B} W_{\alpha}^{B}$ and $\bar{W}_{\dot{\alpha}}^{A} \bar{f}_{A B} \bar{W}^{B \dot{\alpha}}$ are gauge invariant. 
Mutatis mutandis, the vector field transformations define a realization of the complexified chiral gauge group $G^{(+)} \times G^{(-)}$with superfield parameters

$$
\lambda_{ \pm}^{A} \equiv \frac{1}{2}\left(\bar{\Lambda}^{A} \pm \Lambda^{A}\right)
$$

and corresponding gauge transformation functional differential operators

$$
\begin{aligned}
\delta_{A}^{(+)} & =\int d S \delta_{A} \phi^{i} \frac{\delta}{\delta \phi^{i}}+\int d \bar{S} \delta_{A} \bar{\phi}^{\bar{i}} \frac{\delta}{\delta \bar{\phi}^{i}}+\int d V f_{A B C} V^{C} \frac{\delta}{\delta V^{B}} \\
\delta_{A}^{(-)} & =-\int d S \delta_{A} \phi^{i} \frac{\delta}{\delta \phi^{i}}+\int d \bar{S} \delta_{A} \bar{\phi}^{\bar{i}} \frac{\delta}{\delta \bar{\phi}^{i}}+\int d V i(V \operatorname{coth} V)_{A B} \frac{\delta}{\delta V^{B}} .
\end{aligned}
$$

In terms of these variations, the gauge transformations take the form $\delta(\Lambda, \bar{\Lambda})=$ $\lambda_{+}^{A} \delta_{A}^{(+)}+\lambda_{-}^{A} \delta_{A}^{(-)}$. Moreover, the $\delta_{A}^{( \pm)}$variations obey the chiral algebra [14 given by

$$
\begin{aligned}
& {\left[\delta_{A}^{(+)}, \delta_{B}^{(+)}\right]=f_{A B C} \delta_{C}^{(+)}} \\
& {\left[\delta_{A}^{(+)}, \delta_{B}^{(-)}\right]=f_{A B C} \delta_{C}^{(-)}} \\
& {\left[\delta_{A}^{(-)}, \delta_{B}^{(-)}\right]=f_{A B C} \delta_{C}^{(+)} .}
\end{aligned}
$$

As such, the Yang-Mills fields, $V^{A}$, transform in the adjoint representation of the $G^{(+)}$subgroup and provide a non-linear realization of the $G^{(-)}$subgroup. Written in terms of the $\lambda_{ \pm}$superfield parameters, the exponential transformation law of the Yang- Mills fields is

$$
\begin{aligned}
e^{2 V^{\prime}} & =e^{-i \Lambda} e^{2 V} e^{+i \bar{\Lambda}} \\
& =e^{-i\left(\lambda_{+}-\lambda_{-}\right)} e^{2 V} e^{i\left(\lambda_{+}+\lambda_{-}\right)},
\end{aligned}
$$

which in turn yields the infinitesimal transformation laws

$$
\delta_{A}^{(+)} V^{B}=i\left(t^{A}\right)_{B C} V^{C}
$$




$$
\delta_{A}^{(-)} V^{B}=i(V \operatorname{coth} V)_{A B}
$$

in agreement with Eq. (2.20).

Using the $\delta_{A}^{(-)}$variations for the matter fields, the gauge invariant Kähler potential, $K(\phi, \bar{\phi}, V)$, can be constructed [13] 14 from the globally invariant Kähler potential, $K_{0}(\phi, \bar{\phi})$. To achieve this, we define the pure chiral matter field transformation operators $\delta_{A}^{(\phi \pm)}$ as

$$
\begin{aligned}
\delta_{A}^{(\phi+)} & =\int d S \delta_{A} \phi^{i} \frac{\delta}{\delta \phi^{i}}+\int d \bar{S} \delta_{A} \bar{\phi}^{\bar{i}} \frac{\delta}{\delta \bar{\phi}^{i}} \\
\delta_{A}^{(\phi-)} & =-\int d S \delta_{A} \phi^{i} \frac{\delta}{\delta \phi^{i}}+\int d \bar{S} \delta_{A} \bar{\phi}^{\bar{i}} \frac{\delta}{\delta \bar{\phi}^{\bar{i}}} .
\end{aligned}
$$

Then using the commutation relation [14]

$$
\left[\delta(\Lambda, \bar{\Lambda}), e^{i V^{A} \delta_{A}^{(\phi-)}}\right]=e^{i V^{A} \delta_{A}^{(\phi-)}}\left[\lambda_{-}^{B}\left(\tanh \frac{1}{2} V\right)_{B C} \delta_{C}^{(\phi-)}-\lambda_{-}^{B} \delta_{B}^{(\phi-)}\right],
$$

it follows that

$$
K(\phi, \bar{\phi}, V) \equiv e^{i V^{A} \delta_{A}^{(\phi-)}} K_{0}(\phi, \bar{\phi})
$$

is locally gauge invariant

$$
\delta(\Lambda, \bar{\Lambda}) K(\phi, \bar{\phi}, V)=0
$$

provided $K_{0}(\phi, \bar{\phi})$ is globally invariant, $\delta_{A} K_{0}(\phi, \bar{\phi})=0$. A globally invariant Kähler potential can always be found [13] when the group does not contain explicit $U(1)$ factors. Moreover, in that globally noninvariant case, where $\delta_{A} K_{0}(\phi, \bar{\phi})=\bar{F}_{A}(\bar{\phi})+F_{A}(\phi) \neq 0$, a locally invariant form can also be constructed $[13]$.

\section{$2.2 \quad$ Supersymmetry}

In addition to its gauge invariance, the action, Eq. (2.1), is also supersymmetric and Poincaré invariant. These global superspace symmetries are 
represented by superspace differential operators on the superfields, which in turn can be used to construct functional differential operators representing the generators of the symmetries. The supersymmetry transformations are given by

$$
\begin{aligned}
\delta_{\alpha}^{Q} \Phi(x, \theta, \bar{\theta}) & =\left[\frac{\partial}{\partial \theta}+i \sigma^{\mu} \bar{\theta} \partial_{\mu}\right]_{\alpha} \Phi(x, \theta, \bar{\theta}) \\
\delta_{\dot{\alpha}}^{\bar{Q}} \Phi(x, \theta, \bar{\theta}) & =\left[-\frac{\partial}{\partial \bar{\theta}}-i \theta \sigma^{\mu} \partial_{\mu}\right]_{\dot{\alpha}} \Phi(x, \theta, \bar{\theta}),
\end{aligned}
$$

where $\Phi$ is any of the superfields $\phi, \bar{\phi}$ or $V$, while the variation of the fields under space-time translations is given by

$$
\delta_{\mu}^{P} \Phi(x, \theta, \bar{\theta})=\partial_{\mu} \Phi(x, \theta, \bar{\theta}) .
$$

These variations can be combined to form the Ward identity functional differential operator representing the generators of the symmetries. The functional differential operators corresponding to the supersymmetry charges $Q_{\alpha}$ and $\bar{Q}_{\dot{\alpha}}$ are

$$
\begin{aligned}
\delta_{\alpha}^{Q} & =\int d S \delta_{\alpha}^{Q} \phi^{i} \frac{\delta}{\delta \phi^{i}}+\int d \bar{S} \delta_{\alpha}^{Q} \bar{\phi}^{\bar{i}} \frac{\delta}{\delta \bar{\phi}^{i}}+\int d V \delta_{\alpha}^{Q} V \frac{\delta}{\delta V} \\
\delta_{\dot{\alpha}}^{\bar{Q}} & =\int d S \delta_{\dot{\alpha}}^{\bar{Q}} \phi^{i} \frac{\delta}{\delta \phi^{i}}+\int d \bar{S} \delta_{\dot{\alpha}}^{\bar{Q}} \bar{\phi}^{\bar{i}} \frac{\delta}{\delta \bar{\phi}^{i}}+\int d V \delta_{\dot{\alpha}}^{\bar{Q}} V \frac{\delta}{\delta V},
\end{aligned}
$$

while those corresponding to the space-time translation generators $P^{\mu}$ are

$$
\delta_{\mu}^{P}=\int d S \delta_{\mu}^{P} \phi^{i} \frac{\delta}{\delta \phi^{i}}+\int d \bar{S} \delta_{\mu}^{P} \bar{\phi}^{\bar{i}} \frac{\delta}{\delta \bar{\phi}^{\bar{i}}}+\int d V \delta_{\mu}^{P} V \frac{\delta}{\delta V} .
$$

Similar expressions also hold for Lorentz transformations. These variations satisfy an algebra analogous to the one satisfied by the global symmetry generators. For example, while the supersymmetry charges anti-commute to yield the momentum operator,

$$
\left\{Q_{\alpha}, \bar{Q}_{\dot{\alpha}}\right\}=2 \sigma_{\alpha \dot{\alpha}}^{\mu} P_{\mu}
$$


it is readily seen that

$$
\left\{\delta_{\alpha}^{Q}, \delta_{\dot{\alpha}}^{\bar{Q}}\right\}=-2 i \sigma_{\alpha \dot{\alpha}}^{\mu} \delta_{\mu}^{P}
$$

By construction, the action $\Gamma$ is invariant under supersymmetry and spacetime translation transformations so that

$$
\begin{aligned}
\delta_{\alpha}^{Q} \Gamma[\phi, \bar{\phi}, V] & =0 \\
\delta_{\dot{\alpha}}^{\bar{Q}} \Gamma[\phi, \bar{\phi}, V] & =0 \\
\delta_{\mu}^{P} \Gamma[\phi, \bar{\phi}, V] & =0 .
\end{aligned}
$$

The action may also be invariant under R-symmetry or some global internal symmetries. In particular, the generator of R-symmetry transformations is given by

$$
\delta^{R}=\int d S \delta^{R} \phi^{i} \frac{\delta}{\delta \phi^{i}}+\int d \bar{S} \delta^{R} \bar{\phi}^{\bar{i}} \frac{\delta}{\delta \bar{\phi}^{i}}+\int d V \delta^{R} V \frac{\delta}{\delta V},
$$

where the explicit R-symmetry transformations of the fields are defined by

$$
\delta^{R} \Phi(x, \theta, \bar{\theta})=i\left[n_{\Phi}+\theta^{\alpha} \frac{\partial}{\partial \theta^{\alpha}}+\bar{\theta}_{\dot{\alpha}} \frac{\partial}{\partial \bar{\theta}_{\dot{\alpha}}}\right] \Phi(x, \theta, \bar{\theta}),
$$

with $n_{\Phi}$ the R-weight of the superfield $\Phi$. Since the vector superfield is real, its R-weight must be zero: $n_{V}=0$. In general, the R-weight of the chiral superfields, $n_{\phi}$, is arbitrary. In some cases, however, it can be fixed so as to make the superpotential R-invariant. Moreover, as shown in the Appendix, if the chiral superfield transforms non-linearly under the gauge group, its R-weight must be zero: $n_{\phi}=0$. The Weyl spinor supersymmetry charges $Q_{\alpha}$ and $\bar{Q}_{\dot{\alpha}}$ form a representation of the chiral R- symmetry given by

$$
\begin{aligned}
{\left[R, Q_{\alpha}\right] } & =Q_{\alpha} \\
{\left[R, \bar{Q}_{\dot{\alpha}}\right] } & =-\bar{Q}_{\dot{\alpha}} .
\end{aligned}
$$


Likewise, it follows that the Ward identity functional differential operators obey the analogous algebra

$$
\begin{aligned}
& {\left[\delta^{R}, \delta_{\alpha}^{Q}\right]=-i \delta_{\alpha}^{Q}} \\
& {\left[\delta^{R}, \bar{\delta}_{\dot{\alpha}}^{\bar{Q}}\right]=i \bar{\delta}_{\dot{\alpha}}^{\bar{Q}}}
\end{aligned}
$$

Using Noether's theorem, the (non-) conserved currents corresponding to these transformations can be constructed from the action. Since $\Gamma$ is supersymmetric and translation invariant, the corresponding supersymmetry currents, $Q_{\alpha}^{\mu}, \bar{Q}_{\dot{\alpha}}^{\mu}$, and the energy-momentum tensor, $T^{\mu \nu}$, are conserved and satisfy

$$
\begin{aligned}
\partial_{\mu} Q_{\alpha}^{\mu}(x) & =\delta_{\alpha}^{Q}(x) \Gamma \\
\partial_{\mu} \bar{Q}_{\dot{\alpha}}^{\mu}(x) & =\bar{\delta}_{\dot{\alpha}}^{\bar{Q}}(x) \Gamma \\
\partial_{\mu} T^{\mu \nu}(x) & =\delta^{P \nu}(x) \Gamma .
\end{aligned}
$$

Here $\delta_{\alpha}^{Q}(x), \bar{\delta}_{\dot{\alpha}}^{\bar{Q}}(x)$ and $\delta_{\mu}^{P}(x)$ are the local SUSY and translation functional differential operators respectively. The corresponding global transformation functional differential operators, $\delta_{\alpha}^{Q}, \bar{\delta}_{\dot{\alpha}}^{\bar{Q}}, \delta_{\mu}^{P}$, are constructed by integrating the local operators over space-time. Thus, for example, $\delta_{\alpha}^{Q}=\int d^{4} x \delta_{\alpha}^{Q}(x)$, is the global SUSY variation. It follows that the currents of Eq. 2.39 can be modified (improved) by the addition of Belinfante terms or total spacetime divergences of Euler derivatives of the action (contact terms) without alterring the form of the current conservation law or the time independent charges.

Similarly, the R-current can be constructed via Noether's theorem as

$$
\partial_{\mu} R^{\mu}(x)=\delta^{R}(x) \Gamma-i S_{R}(x),
$$


where $S_{R}(x)$ describes the explicit R-symmetry breaking of the action. Integrating this equation over space-time gives

$$
\delta^{R} \Gamma[\phi, \bar{\phi}, V]=i \int d^{4} x S_{R}(x),
$$

which constitutes the global R Ward identity.

The R-current so defined can be extended so as to form an entire superfield with $R^{\mu}(x)$ as its lowest component. It is this multiplet with appropriately defined improved supersymmetry currents and energy-momentum tensor which constitutes the supercurrent. By construction the supercurrent contains a (non-) conserved R-symmetry current $R^{\mu}(x)$ as the lowest component with conserved supersymmetry currents, $Q_{\alpha}^{\mu}(x), \bar{Q}_{\dot{\alpha}}^{\mu}(x)$, and symmetric energy-momentum tensor, $T^{\mu \nu}(x)$, in higher components [9] 15] 16]. 


\section{The Supercurrent}

In general, the generators of global symmetry transformations can be obtained from the symmetry currents using Noether's theorem. For the superconformal symmetries, all superconformal currents can be gleaned from the supercurrent [15][16]. The supercurrent is just the superfield of currents whose first component is given by the R-symmetry current and which, moreover, contains the supersymmetry currents and the improved energymomentum tensor as higher dimension components. It has been shown on very general grounds [15] [16] that the real supercurrent, $V_{\alpha \dot{\alpha}}=\frac{1}{2} \sigma_{\alpha \dot{\alpha}}^{\mu} V_{\mu}$, must satisfy a general set of spinor derivative (trace) equations of the form

$$
\begin{aligned}
\bar{D}^{\dot{\alpha}} V_{\alpha \dot{\alpha}} & =-2 \hat{\delta}_{\alpha} \Gamma+B_{\alpha}-2 D_{\alpha} S \\
D^{\alpha} V_{\alpha \dot{\alpha}} & =-2 \hat{\bar{\delta}}_{\dot{\alpha}} \Gamma+\bar{B}_{\dot{\alpha}}-2 \bar{D}_{\dot{\alpha}} \bar{S} .
\end{aligned}
$$

The $B_{\alpha}$ and $\bar{B}_{\dot{\alpha}}$ are restricted to obey $D^{\alpha} B_{\alpha}=\bar{D}_{\dot{\alpha}} \bar{B}^{\dot{\alpha}}$ while $(\bar{S}) S$ is a (anti-) chiral superfield, $\left(D^{\alpha} \bar{S}=0\right) \bar{D}_{\dot{\alpha}} S=0$. In order for $V_{\alpha \dot{\alpha}}$ to contain a conserved energy-momentum tensor $T^{\mu \nu}$, and supersymmetry currents $Q_{\alpha}^{\mu}$ and $\bar{Q}_{\dot{\alpha}}^{\mu}$, it must be that the symmetry breaking terms $\left(\bar{B}_{\dot{\alpha}}\right) B_{\alpha}$ and $(\bar{S}) S$ cannot both be non-zero simultaneously. The local superspace Ward identity functional differential operators, $\hat{\delta}_{\alpha}, \hat{\bar{\delta}}_{\dot{\alpha}}$, are defined as

$$
\begin{aligned}
\hat{\bar{\delta}}_{\dot{\alpha}} \equiv & n_{\phi} \bar{D}_{\dot{\alpha}}\left(\bar{\phi}^{\bar{i}} \frac{\delta}{\delta \bar{\phi}^{i}}\right)+2\left(\bar{D}_{\dot{\alpha}} \bar{\phi}^{\bar{i}}\right) \frac{\delta}{\delta \bar{\phi}^{i}} \\
& -2\left(D D \bar{D}_{\dot{\alpha}} V^{A}\right) \frac{\delta}{\delta V^{A}}+2\left(\bar{D}_{\dot{\alpha}} V^{A}\right) D D \frac{\delta}{\delta V^{A}} \\
& +2 D^{\alpha}\left[\bar{D}_{\dot{\alpha}} V^{D} D_{\alpha} V^{A}\left(\ell_{A B, D} \ell_{B C}^{-1}+\bar{\ell}_{D B},_{A} \bar{\ell}_{B C}^{-1}\right) \frac{\delta}{\delta V^{C}}\right] \\
\hat{\delta}_{\alpha} \equiv & n_{\phi} D_{\alpha}\left(\phi^{i} \frac{\delta}{\delta \phi^{i}}\right)+2\left(D_{\alpha} \phi^{i}\right) \frac{\delta}{\delta \phi^{i}} \\
& -2\left(\bar{D} \bar{D} D_{\alpha} V^{A}\right) \frac{\delta}{\delta V^{A}}+2\left(D_{\alpha} V^{A}\right) \bar{D} \bar{D} \frac{\delta}{\delta V^{A}}
\end{aligned}
$$




$$
+2 \bar{D}_{\dot{\alpha}}\left[D_{\alpha} V^{D} \bar{D}^{\dot{\alpha}} V^{A}\left(\ell_{A B},_{D} \ell_{B C}^{-1}+\bar{\ell}_{D B, A} \bar{\ell}_{B C}^{-1}\right) \frac{\delta}{\delta V^{C}}\right] .
$$

Note that when restricted to Abelian gauge fields, the last lines on the right hand side of each equation vanishes. The form of these variations is such that all the superconformal transformations can be secured by acting on them with appropriate spinor derivatives and then constructing their various space-time moments. In particular, defining the local variation

$$
\hat{\delta} \equiv i\left(D^{\alpha} \hat{\delta}_{\alpha}-\bar{D}_{\dot{\alpha}} \hat{\bar{\delta}}^{\dot{\alpha}}\right)
$$

then its space-time integral

$$
\delta=\int d^{4} x \hat{\delta}
$$

forms the superfield containing the (previously defined) R symmetry, supersymmetry and space-time translation functional differential operators, $\delta^{R}$, $\delta_{\alpha}^{Q}, \bar{\delta}_{\dot{\alpha}}^{\bar{Q}}$ and $\delta_{\mu}^{P}$, as

$$
\delta=\delta^{R}-i \theta^{\alpha} \delta_{\alpha}^{Q}+i \bar{\theta}_{\dot{\alpha}} \bar{\delta}^{\bar{Q} \dot{\alpha}}-2 \theta \sigma^{\mu} \bar{\theta} \delta_{\mu}^{P}
$$

Note that alternate forms for $\hat{\delta}_{\alpha}$ and $\hat{\bar{\delta}}_{\dot{\alpha}}$ can also be defined by adding various terms which take the form of additional total derivatives of contact terms (improvements) or have the effect of changing the relation of these variations to the conformal transformations [16]. The conservation of the supersymmetry currents, $Q_{\alpha}^{\mu}, \bar{Q}_{\dot{\alpha}}^{\mu}$, and the energy-momentum tensor, $T^{\mu \nu}$, Eq. (2.39), follows from Eq. (3.1) provided either (or both) $S$ or $B$ to vanish, which alternative being a model dependent question [15] [16].

Applying the spinor derivative construction of Eq. (3.3) to Eq. (3.1) yields the space-time divergence equation for the supercurrent

$$
\partial^{\mu} V_{\mu}=\frac{1}{2 i}\left\{D^{\alpha}, \bar{D}^{\dot{\alpha}}\right\} V_{\alpha \dot{\alpha}}
$$




$$
=\delta \Gamma-i(\bar{D} \bar{D} \bar{S}-D D S)
$$

The $\theta, \bar{\theta}$ independent component of this equation gives the R-current Ward identity

$$
\partial^{\mu} R_{\mu}=\delta^{R}(x) \Gamma-\left.i(\bar{D} \bar{D} \bar{S}-D D S)\right|_{\theta=\bar{\theta}=0} .
$$

If $S \neq 0$, the $R$-symmetry is explicitly broken. Note that in such a case, in order for the supercurrent to contain a conserved supersymmetry current and energy-momentum tensor, it is required that $B=0$. The construction of all the superconformal currents along with their associated Ward identities and anomalies is detailed in references [15] [16]. Besides the R-symmetry current constructed above as the $\theta, \bar{\theta}$ independent component of the supercurrent itself,

$$
R_{\mu}=\left.V_{\mu}\right|_{\theta=\bar{\theta}=0}
$$

the supersymmetry currents and the energy-momentum tensor can similarly be constructed as the $\theta, \bar{\theta}$ independent components of certain combinations of spinor derivatives acting on the supercurrent as:

$$
\begin{aligned}
Q_{\mu \alpha} & =\left.i\left(D_{\alpha} V_{\mu}-\left(\sigma_{\mu} \bar{\sigma}^{\nu} D\right)_{\alpha} V_{\nu}\right)\right|_{\theta=\bar{\theta}=0} \\
\bar{Q}_{\mu \dot{\alpha}} & =-\left.i\left(\bar{D}_{\dot{\alpha}} V_{\mu}-\left(\bar{\sigma}_{\mu} \sigma^{\nu} \bar{D}\right)_{\dot{\alpha}} V_{\nu}\right)\right|_{\theta=\bar{\theta}=0} \\
T_{\mu \nu} & =-\left.\frac{1}{16}\left(V_{\mu \nu}+V_{\nu \mu}-2 g_{\mu \nu} V_{\rho}^{\rho}\right)\right|_{\theta=\bar{\theta}=0},
\end{aligned}
$$

where the superfield $V_{\mu \nu}$ is defined as

$$
V_{\mu \nu}=\left(D \sigma_{\mu} \bar{D}-\bar{D} \bar{\sigma}_{\mu} D\right) V_{\nu}
$$

The remaining superconformal currents and angular momentum tensor can be constructed as space-time moments of Eq. 3.9. For example the dilatation current is given by $D_{\mu}=x^{\nu} T_{\mu \nu}$. 
Given an action $\Gamma$ and the variations $\hat{\delta}_{\alpha}$ and $\hat{\bar{\delta}}_{\dot{\alpha}}$, the $V_{\alpha \dot{\alpha}}, B_{\alpha}, \bar{B}_{\dot{\alpha}}, S$ and $\bar{S}$ are constructed so as to satisfy the trace equations (3.1). Towards this end, it is necessary to use the field equations for the matter and Yang-Mills superfields. Functionally differentiating the action of Eq. (2.1) with respect to the chiral and anti-chiral matter fields yields

$$
\begin{aligned}
\frac{\delta \Gamma}{\delta \phi^{i}} & =-\frac{1}{4} \bar{D} \bar{D} K_{, i}+P_{, i}-2 f_{A B, i} W^{A} W^{B} \\
\frac{\delta \Gamma}{\delta \bar{\phi}^{i}} & =-\frac{1}{4} D D K_{, \bar{i}}+\bar{P}_{, \bar{i}}-2 \bar{f}_{A B, \bar{i}} \bar{W}^{A} \bar{W}^{B} .
\end{aligned}
$$

A useful form of the Yang-Mills field equations [18] is obtained by introducing the gauge covariant spinor derivatives $\mathcal{D}_{\alpha}$ and $\overline{\mathcal{D}}_{\dot{\alpha}}$ for the chiral field strength spinors as,

$$
\begin{aligned}
\mathcal{D}^{\alpha} W_{\beta} & \equiv e^{-2 V} D^{\alpha}\left[e^{2 V} W_{\beta} e^{-2 V}\right] e^{2 V} \\
& =D^{\alpha} W_{\beta}+\Omega^{\alpha} W_{\beta}+W_{\beta} \Omega^{\alpha} \\
\overline{\mathcal{D}}_{\dot{\alpha}} \bar{W}^{\dot{\beta}} & \equiv e^{2 V} \bar{D}_{\dot{\alpha}}\left[e^{-2 V} \bar{W}^{\dot{\beta}} e^{2 V}\right] e^{-2 V} \\
& =\bar{D}_{\dot{\alpha}} \dot{W}^{\dot{\beta}}+\bar{\Omega}_{\dot{\alpha}} \bar{W}^{\dot{\beta}}+\bar{W}^{\dot{\beta}} \bar{\Omega}_{\dot{\alpha}},
\end{aligned}
$$

with

$$
\begin{aligned}
& \Omega_{\alpha} \equiv e^{-2 V} D_{\alpha} e^{2 V}=\left(D_{\alpha} V^{A} \ell_{A B}\right) t^{B} \equiv \Omega_{\alpha}^{B} t^{B} \\
& \bar{\Omega}_{\dot{\alpha}} \equiv e^{2 V} D_{\dot{\alpha}} e^{-2 V}=\left(\bar{D}_{\dot{\alpha}} V^{A} \bar{\ell}_{A B}\right) t^{B} \equiv \bar{\Omega}_{\dot{\alpha}}^{B} t^{B} .
\end{aligned}
$$

Alternatively, these covariant derivatives can be written as

$$
\begin{aligned}
\left(\mathcal{D}^{\alpha} W_{\beta}\right)^{C} & =\left(e^{-2 V}\right)_{C B} D^{\alpha}\left[\left(e^{2 V}\right)_{B A} W_{\beta}^{A}\right] \\
& =D^{\alpha} W_{\beta}^{C}+i f_{C B A} \Omega_{B}^{\alpha} W_{\beta}^{A}
\end{aligned}
$$




$$
\begin{aligned}
\left(\overline{\mathcal{D}}_{\dot{\alpha}} \bar{W}^{\dot{\beta}}\right)^{C} & =\left(e^{2 V}\right)_{C B} \bar{D}_{\dot{\alpha}}\left[\left(e^{-2 V}\right)_{B A} \bar{W}^{A \dot{\beta}}\right] \\
& =\bar{D}_{\dot{\alpha}} \bar{W}^{C \dot{\beta}}+i f_{C B A} \bar{\Omega}_{\dot{\alpha}}^{B} \bar{W}^{A \dot{\beta}} .
\end{aligned}
$$

The field equations for the gauge fields can then be cast as

$$
\begin{aligned}
\frac{\delta \Gamma}{\delta V^{A}} & =-\ell_{A B}\left(\mathcal{D}^{\alpha} F_{\alpha}\right)^{B}-\bar{\ell}_{A B}\left(\overline{\mathcal{D}}_{\dot{\alpha}} \bar{F}^{\dot{\alpha}}\right)^{B} \\
& =-\ell_{A B}\left(\mathcal{D}^{\alpha} F_{\alpha}\right)^{B}+\left(\overline{\mathcal{D}}_{\dot{\alpha}} \bar{F}^{\dot{\alpha}}\right)^{B} \ell_{B A},
\end{aligned}
$$

where we have introduced the auxiliary field strength spinors $F_{\alpha}$ and $\bar{F}_{\dot{\alpha}}$ defined as $F^{\alpha} \equiv t^{A} f_{A B} W_{\alpha}^{B}$ and $\bar{F}_{\dot{\alpha}} \equiv t^{A} \bar{f}_{A B} \bar{W}_{\dot{\alpha}}^{B}$. In addition to these dynamical relations, the field strength spinors also satisfy the Bianchi identities

$$
\mathcal{D}^{\alpha} W_{\alpha}=-e^{-2 V}\left(\overline{\mathcal{D}}_{\dot{\alpha}} \bar{W}^{\dot{\alpha}}\right) e^{2 V}
$$

which can alternatively be written as

$$
\left(\overline{\mathcal{D}}_{\dot{\alpha}} \bar{W}^{\dot{\alpha}}\right)^{A}=-\left(e^{2 V}\right)_{A B}\left(\mathcal{D}^{\alpha} W_{\alpha}\right)^{B}
$$

or in further detail

$$
\bar{D}_{\dot{\alpha}}\left[\left(e^{-2 V}\right)_{A B} \bar{W}^{B \dot{\alpha}}\right]=-\left(e^{-2 V}\right)_{A C} D^{\alpha}\left[\left(e^{2 V}\right)_{C B} W_{\alpha}^{B}\right] .
$$

Application of the Ward identity operator $\hat{\bar{\delta}}_{\dot{\alpha}}$ to the general action of Eq. (2.1) and exploiting the field equations (3.11), 3.15 along with the Bianchi identity (3.18), the supercurrent trace equation (3.1) is seen to be satisfied with the supercurrent identified as

$$
\begin{aligned}
V_{\alpha \dot{\alpha}}= & 16\left[\bar{W}_{\dot{\alpha}}^{A}\left(e^{2 V}\right)_{A B} f_{B C} W_{\alpha}^{C}-W_{\alpha}^{A}\left(e^{-2 V}\right)_{A B} \bar{f}_{B C} \bar{W}_{\dot{\alpha}}^{C}\right] \\
& -\frac{2}{3}\left[D_{\alpha}, \bar{D}_{\dot{\alpha}}\right] K+2 K,_{i \bar{i}} \mathcal{D}_{\alpha} \phi^{i} \overline{\mathcal{D}}_{\dot{\alpha}} \bar{\phi}^{\bar{i}}
\end{aligned}
$$


Here we have introduced the gauge covariant spinor derivatives for the matter fields defined as

$$
\begin{aligned}
\mathcal{D}_{\alpha} \phi^{i} & \equiv D_{\alpha} \phi^{i}-i \Omega_{\alpha}^{B} A_{B}^{i}(\phi) \\
& =D_{\alpha} \phi^{i}-i D_{\alpha} V^{A} \ell_{A B} A_{B}^{i}(\phi) \\
\overline{\mathcal{D}}_{\dot{\alpha}} \bar{\phi}^{\bar{i}} & \equiv \bar{D}_{\dot{\alpha}} \bar{\phi}^{\bar{i}}-i \bar{\Omega}_{\dot{\alpha}}^{B} \bar{A}_{B}^{\bar{i}}(\bar{\phi}) \\
& =\bar{D}_{\dot{\alpha}} \bar{\phi}^{\bar{i}}-i \bar{D}_{\dot{\alpha}} V^{A} \bar{\ell}_{A B} \bar{A}_{B}^{\bar{i}}(\bar{\phi}),
\end{aligned}
$$

which have the gauge variations

$$
\begin{aligned}
\delta(\Lambda, \bar{\Lambda}) \mathcal{D}_{\alpha} \phi^{i} & =\Lambda^{A} A_{A}^{i},{ }_{j}(\phi) \mathcal{D}_{\alpha} \phi^{j} \\
\delta(\Lambda, \bar{\Lambda}) \overline{\mathcal{D}}_{\dot{\alpha}} \bar{\phi}^{\bar{i}} & =\bar{\Lambda}^{A} \bar{A}_{A}^{\bar{i}}, \bar{j}(\bar{\phi}) \overline{\mathcal{D}}_{\dot{\alpha}} \bar{\phi}^{\bar{j}} .
\end{aligned}
$$

Note that $V_{\alpha \dot{\alpha}}$ is manifestly real and gauge invariant. In addition, one finds explicitly that $\bar{B}_{\dot{\alpha}}=0$, while the anti-chiral breaking terms have the form

$$
\begin{aligned}
\bar{S}= & -\frac{1}{4} D D\left(-\frac{2}{3} K-n_{\phi}\left(\bar{\phi}^{\bar{i}} K, \bar{i}\right)\right)+8 n_{\phi} \bar{\phi}^{\bar{i}} \bar{W}_{\dot{\beta}}^{A} \bar{f}_{A B, \bar{i}} \bar{W}^{B \dot{\beta}} \\
& -\left(2 \bar{P}+n_{\phi} \bar{\phi}^{\bar{i}} \bar{P}_{, \bar{i}}\right) .
\end{aligned}
$$

Since $\bar{S} \neq 0$, the R symmetry is, in general, explicitly broken.

Using this general form of the supercurrent and its associated Ward identity, various special cases can be considered. First of all, the form for $V_{\alpha \dot{\alpha}}$ and $\bar{S}$ reduce to their previously established values [16],

$$
\begin{aligned}
V_{\alpha \dot{\alpha}} & =32 \bar{W}_{\dot{\alpha}} e^{2 V^{A} t^{A}} W_{\alpha}-\frac{2}{3}\left[D_{\alpha}, \bar{D}_{\dot{\alpha}}\right]\left(\phi e^{2 V^{A} T^{A}} \bar{\phi}\right)+2 \mathcal{D}_{\alpha} \phi e^{2 V^{A} T^{A}} \overline{\mathcal{D}}_{\dot{\alpha}} \bar{\phi} \\
\bar{S} & =\frac{\left(2+3 n_{\phi}\right)}{12} D D\left(\phi e^{2 V^{A} T^{A}} \bar{\phi}\right)-\left(2 \bar{P}+n_{\phi} \bar{\phi}^{\bar{i}} \bar{P}_{, \bar{i}}\right)
\end{aligned}
$$

when the model is restricted to be renormalizable so that $K=\phi e^{2 V \cdot T} \bar{\phi}$ and $P$ is at most trilinear in $\phi$ while $f_{A B}=\delta_{A B}$. For conformal R-weight $n_{\phi}=-\frac{2}{3}$ 
and no mass or linear terms in $P$, the breaking terms vanish, $S=0$, and the R-current is conserved.

For the particular case when the gauge symmetry is non- linearly realized on the chiral matter fields with a vanishing superpotential, it follows (see Appendix) that the $\phi$ field R-weight must be zero: $n_{\phi}=0$. The supercurrent can then be cast as

$$
V_{\alpha \dot{\alpha}}=16\left[\bar{W}_{\dot{\alpha}}^{A}\left(e^{2 V}\right)_{A B} f_{B C} W_{\alpha}^{C}-W_{\alpha}^{A}\left(e^{-2 V}\right)_{A B} \bar{f}_{B C} \bar{W}_{\dot{\alpha}}^{C}\right]+2 \mathcal{D}_{\alpha} \phi^{i} K,_{i \bar{i}} \overline{\mathcal{D}}_{\dot{\alpha}} \bar{\phi}^{\bar{i}}
$$

with $\bar{S}=0$ and $\bar{B}_{\dot{\alpha}}=D D \bar{D}_{\dot{\alpha}} K$. Since $\bar{S}=0$, this form of the supercurrent not only leads to a conserved supersymmetry current and energy-momentum tensor, but also to a conserved R-symmetry current.

When the chiral matter fields form a linear representation of the gauge group, the axial R-weight $n_{\phi}$ is arbitrary. If, however, the superpotential and prepotential are R-invariant so that

$$
\begin{aligned}
2 \bar{P}_{+} n_{\phi} \bar{\phi}^{\bar{i}} \bar{P}_{, \bar{i}} & =0, \\
\bar{f}_{A B}=f_{A B} & =\delta_{A B},
\end{aligned}
$$

while the gauged Kähler potential possesses an additional global, axial $U_{A}(1)$ symmetry, so that

$$
\phi^{i} K,_{i}-\bar{\phi}^{\bar{i}} K,_{i}=0
$$

then the $\bar{S}$ breaking term can again be traded for a $B$ breaking with a suitable modification of the supercurrent. So doing, we find

$$
\begin{aligned}
V_{\alpha \dot{\alpha}}= & 32 \bar{W}_{\dot{\alpha}} e^{2 V} W_{\alpha} \\
& +2 \mathcal{D}_{\alpha} \phi^{i} K,_{i \bar{i}} \overline{\mathcal{D}}_{\dot{\alpha}} \bar{\phi}^{\bar{\phi}}+\frac{n_{\phi}}{2}\left[D_{\alpha}, \bar{D}_{\dot{\alpha}}\right]\left(\phi^{i} K,_{i}+\bar{\phi}^{i} K, \bar{i}\right)
\end{aligned}
$$




$$
\begin{aligned}
\bar{S} & =0 \\
\bar{B}_{\dot{\alpha}} & =-\frac{1}{4} D D \bar{D}_{\dot{\alpha}}\left[-4 K-3 n_{\phi}\left(\phi^{i} K_{, i}+\bar{\phi}^{\bar{i}} K, \bar{i}\right)\right] .
\end{aligned}
$$

The quantization of the gauge models requires the introduction of gauge fixing and Fadeev-Popov terms to the Lagrangian [16] 18. With their inclusion, the action ceases to be gauge invariant but becomes BRS invariant. A detailed account of the supercurrent construction in renormalizable models with BRS invariance can be found in the literature [16]. When the quantum corrections are taken into account, the divergence of the R-current, the $\gamma$-trace of the supersymmetry current and the Lorentz trace of the energymomentum tensor are anomalous with the renormalization group $\beta$ function as the anomaly coefficient 91015 [19. The nature of these radiative corrections for the renormalizable $\mathrm{N}=1$ SUSY models has been investigated and reviewed [16] 20] 21] 22]. For certain gauge models, the $\beta$ function has been shown [5] [6] or argued [23] to vanish identically at a superconformal fixed point. This, in turn, fixes the R-weights.

In all of the above, discussion has been restricted to the case of linearly realized supersymmetry, while the gauge symmetry was allowed to be realized either lineraly or non-linearly. For completeness, let us recall the case of a non-linearly realized supersymmetry. Indeed in the absence of explicit breakings, if supersymmetry is to be realized in nature, it must be as a spontaneously broken symmetry. At high energy, the short distance behavior of the theory will be unaffected by the soft spontaneous SUSY breaking of the ground state. The structure of the supercurrent will be identical to the unbroken case. At low energy, the spontaneously broken SUSY can be described by the Akulov-Volkov effective Lagrangian [24]. For this model, a supercurrent has also been constructed [25]. It again contains the conserved 
R-current as the lowest component and conserved supersymmetry current and the energy-monentum tensor as higher components. In this case, the R-current is simply given by $R^{\mu}=-2 \kappa^{2} \lambda \sigma_{\nu} \bar{\lambda} T^{\nu \mu}$, where $\lambda$ is the Goldstino field and $\kappa$ is its decay constant and $T^{\mu \nu}$ is the improved energy-momentum tensor.

This work was supported in part by the U.S. Department of Energy under grant DE-AC02-76ER01428 (Task B). 


\section{Appendix A}

In this appendix, we show that the R-weight, $n_{\phi}$, of any chiral superfield transforming non-linearly under an internal symmetry transformation must vanish: $n_{\phi}=0$. This demonstration employs the algebra

$$
\begin{aligned}
{\left[T_{A}, T_{B}\right] } & =i f_{A B C} T_{C} \\
{\left[R, T_{A}\right] } & =0
\end{aligned}
$$

along with the chiral field transformation laws

$$
\begin{aligned}
\delta_{A} \phi^{i} & =\frac{1}{i}\left[T_{A}, \phi^{i}\right]=A_{A}^{i}(\phi) \\
\delta^{R} \phi^{i} & =\frac{1}{i}\left[R, \phi^{i}\right]=i\left(n_{\phi}+\theta^{\alpha} \frac{\partial}{\partial \theta^{\alpha}}+\bar{\theta}_{\dot{\alpha}} \frac{\partial}{\partial \bar{\theta}_{\dot{\alpha}}}\right) \phi^{i}
\end{aligned}
$$

Using these relations, the Jacobi identity

$$
0=\left[\left[R, T_{A}\right], \phi^{i}\right]+\left[\left[\phi^{i}, R\right], T_{A}\right]+\left[\left[T_{A}, \phi^{i}\right], R\right]
$$

reduces to

$$
0=n_{\phi}\left(A_{A}^{i}-A_{A, j}^{i} \phi^{j}\right)
$$

For non-linear realizations

$$
A_{A, j}^{i} \phi^{j} \neq A_{A}^{i}
$$

and hence we conclude that $n_{\phi}=0$. 


\section{References}

[1] R. Haag, J. Lopuszanski and M. Sohnius, Nucl. Phys. B88 (1975) 257;

S. Coleman and J. Mandula, Phys. Rev. 159 (1967) 1251.

[2] See, for example, M. Green, J. Schwarz and E. Witten, Superstring Theory (Cambridge, New York, 1987).

[3] K. Fujikawa and W. Lang Nucl. Phys. B88 (1975) 61.

[4] For reviews, see L.E. Ibánẽz and G.G. Ross in Perspectives on Higgs Physics ed. by G.L. Kane (World Scientific, Singapore, 1993), 229; H.E. Haber and G.L. Kane, Phys. Rep. 117 (1985) 75; H.P. Nilles, Phys. Rep. 110 (1984) 1.

[5] N. Seiberg, Phys. Lett. B318 (1993) 469; B206 (1988) 75.

[6] N. Seiberg and E. Witten, Nucl. Phys. B426 (1994) 19; B431 (1994) 484.

[7] E. Witten and D. Olive, Phys. Lett. B78 (1978) 97.

[8] I. Affleck, M. Dine and N. Seiberg, Nucl. Phys. 256 (1985) 557; A. Nelson and N. Seiberg, Nucl. Phys. B416 (1994) 46; J. Bagger, E. Poppitz and L. Randall, Nucl. Phys. B426 (1994) 3.

[9] S. Ferrara and B. Zumino, Nucl. Phys. B87 (1975) 207.

[10] B. Zumino, Phys. Lett. B87 (1979) 203.

[11] For SUSY notations and conventions, see, J. Wess and J. Bagger, Supersymmetry and Supergravity, (Princeton University Press, Princeton 1992). 
[12] L. Alvarez-Gaumé and D.Z. Freedman, Comm. Math. Phys. 80 (1981) 443; J. Bagger and E. Witten, Phys. Lett. B118 (1982) 103; for a review, see W. Buchmüller and W. Lerche, Ann. of Phys. 175 (1987) 159.

[13] T.E. Clark and S.T. Love, Nucl. Phys. B232 (1984) 306.

[14] T.E. Clark and S.T. Love, Nucl. Phys. B254 (1985) 569.

[15] T.E. Clark, O. Piguet and K. Sibold, Nucl. Phys. B143 (1978) 445; B172 (1980) 201.

[16] O. Piguet and K. Sibold, Renormalized Supersymmetry (Birkhäuser, Boston, 1986) and references therein.

[17] See also, P. West, Introduction to Supersymmetry and Supergravity (World Scientific, Singapore, 1986) and references therein.

[18] S. Ferrara and O. Piguet, Nucl. Phys. B93 (1975) 261.

[19] S.J. Gates, M.T. Grisaru, M. Roček and W. Siegel, Superspace (Benjamin-Cummings, Reading, MA, 1983).

[20] M.T. Grisaru, B. Milewski and D. Zanon, Nucl. Phys. B266 (1986) 589.

[21] M.A. Shifman and A.I. Vainshtein, Nucl. Phys. B277 (1986) 456.

[22] K. Konishi, Phys. Lett. B135 (1984) 439.

[23] N. Seiberg, Nucl. Phys. B435 (1995) 129.

[24] V.P. Akulov and D.V. Volkov, Pis'ma Zh. Eksp. Teor. Fiz. 16 (1972) 621 [JETP Lett. 16 (1972) 438].

[25] T.E. Clark and S.T. Love, Phys. Rev. D39 (1989) 2391. 